\title{
Conhecimento de médicos e enfermeiros obstetras sobre a prevenção dos defeitos no tubo neural
}

\author{
Knowledge of physicians and obstetric nurses \\ about the prevention of neural tube defects
}

\author{
Ricardo Campelo da Conceição ${ }^{1}$ \\ Marcella Amaral Horta Barbosa ${ }^{2}$ \\ Leonardo Lima Dornela ${ }^{2}$ \\ Plínio Santos Ramos ${ }^{3}$ \\ Didier Silveira Castellano Filho ${ }^{1}$ \\ Djalma Rabelo Ricardo ${ }^{4}$ \\ Adriano Almeida Calado ${ }^{5}$ \\ José Murillo Bastos Netto ${ }^{2}$
}

${ }^{1}$ Faculdade de Ciências Médicas e da Saúde de Juiz de Fora. BR $040 \mathrm{Km} 796$, Salvaterra. 36045-410 Juiz de Fora MG.

jmurillo@uropedjf.com.br

${ }^{2}$ Faculdade de Enfermagem,

Universidade Federal de

Juiz de Fora (UFJF).

${ }^{3}$ Hospital e Maternidade

Therezinha de Jesus.

${ }^{4}$ Universidade Gama Filho.

${ }^{5}$ Faculdade de Medicina,

Universidade de

Pernambuco (UPE).
Abstract The scope of this study was to evaluate the knowledge of obstetricians and obstetric nurses about folic acid in the prevention of neural tube defects (NTD). A cross-sectional study was conducted in which 118 volunteers (95 physicians and 23 nurses) answered a questionnaire with 21 questions about prevention of NTD. The data were analyzed according to the reported knowledge, the recommendations made by the Brazilian Ministry of Health $(\mathrm{MOH})$ and the scientific evidence (SE) available in the literature on the topic. The knowledge based on the SE and recommendations of $\mathrm{MOH}$ was different (4.64 \pm 0.20 vs. $5.55 \pm 0.15, p<0.001$, Mean \pm SEM). There was no difference between the knowledge of respondents compared to their training, the time spent in prenatal care and between the two classes of professionals evaluated. There were differences between the reported knowledge and that based on SE (6.76 $\pm 0.18 v$ vs. $4.64 \pm 0.15, p<0.001)$ and based on the $\mathrm{MOH}$ recommendations (6.76 \pm 0.18 vs. $5.55 \pm 0.20, p<0.001)$. Thus, the conclusion reached was that $94.1 \%$ of those evaluated reported knowing the importance of folic acid in preventing NTD, though $64.2 \%$ reported that they did not know when to begin administration of the supplement.

Key words Folic acid, Neural tube closure defects, Prevention
Resumo Foi avaliado o nível de conhecimento de médicos e enfermeiros obstetras sobre a utilização de ácido fólico (AF) para a prevenção dos defeitos do fechamento do tubo neural (DFTN). Estudo descritivo transversal no qual 118 voluntários (95 médicos e 23 enfermeiros), responderam um questionário com 21 perguntas sobre a prevenção dos DFTN. Os dados foram analisados em função do conhecimento relatado, das recomendações preconizadas pelo Ministério da Saúde (MS) e das evidências cientificas (EC) disponiveis sobre o assunto investigado. O nivel de conhecimento baseado nas EC e nas recomendações do MS foi diferente (4,64 $\pm 0,20$ vs 5,55 \pm 0,15; $p<0,001$; Média \pm $E P M)$. Não houve diferença entre o nível de conhecimento quando comparado à formação dos mesmos, ao tempo que realizam atendimento prénatal e entre as duas classes de profissionais avaliados. Houve diferença entre o nivel de conhecimento relatado e o baseado nas $E C(6,76 \pm 0,18$ vs 4,64 \pm $0,15 ; p<0,001)$ e o baseado nas recomendações do MS (6,76 $\pm 0,18$ vs 5,55 $\pm 0,20 ; p<0,001), 94,1 \%$ dos profissionais conhecem o papel do ácido fólico, contudo, $64,2 \%$ relataram não saber quando iniciar a suplementação no pré-natal. Frente ao exposto foi verificado que a grande maioria dos avaliados relatou conhecer a importância do AF na prevenção do DFTN, porém, existe ainda desconhecimento sobre o assunto investigado.

Palavras-chave Ácido fólico, Defeitos do fechamento do tubo neural, Prevenção 


\section{Introdução}

Os defeitos do fechamento do tubo neural (DFTN) estão entre as malformações congênitas mais frequentes, sendo que, a anencefalia e o disrafismo espinhal representam 95\% dos casos, e a encefalocele, $5 \%{ }^{1,2}$.

Nos Estados Unidos, o gasto médio estimado com uma criança nascida com DFTN, por toda a vida, é de aproximadamente US\$ 294.000,003. Embora varie consideravelmente nas diversas regiões geográficas, a incidência dos DFTN, se situa em torno de 1/1.000 nascidos vi$\operatorname{vos}^{3,4}$ e cerca de 95\% das mulheres com filhos afetados por DFTN não apresentam antecedentes pessoais ou familiares ${ }^{5,6}$.

$\mathrm{O}$ ácido fólico (AF) é considerado um nutriente essencial ao ser humano, atuando como coenzima no metabolismo de aminoácidos, purinas, pirimidinas e ácidos nucléicos, sendo vital para a divisão celular e para a síntese de proteínas. Atualmente a deficiência de AF é considerada o mais importante fator de risco para o desenvolvimento dos DFTN $^{7,8}$.

As evidencias científicas (EC) recomendam a suplementação de $0,4 \mathrm{mg} /$ dia de AF para a prevenção dos DFTN, em adendo recomendam a suplementação de $4 \mathrm{mg}$ /dia para as pacientes com um histórico de DFTN em gestação anterior ${ }^{5,8-12}$. Já o Ministério da Saúde (MS) recomenda que mulheres com possibilidade de engravidar devem fazer uso de $5 \mathrm{mg} /$ dia de AF, iniciando 60 a 90 dias antes da concepção e mantendo durante toda a gestação. Face ao exposto parece que existe uma divergência entre a comunidade científica, aqui representada pelas EC, e as recomendações do MS na utilização do AF para a prevenção dos DFTN, portanto, o objetivo do presente estudo foi avaliar o nível de conhecimento de médicos e enfermeiros obstetras sobre a utilização do AF na prevenção dos DFTN.

\section{Métodos}

\section{Amostra}

Foram avaliados 118 profissionais de saúde, entre médicos obstetras (95) e enfermeiros obstetras (23) correspondendo a $50 \%$ e $72 \%$, respectivamente, de todos os profissionais atuantes na cidade de Juiz de Fora (MG), município polo da Macrorregião Sudeste em Saúde, que conta com uma população referenciada de 1.577.484 habitantes, entre dezembro de 2008 a julho de
2009. Os profissionais tinham idades entre $23 \mathrm{e}$ 78 anos e média de $44 \pm 12$ anos (média $\pm \mathrm{dp}$ ); eram formados em média há 18 anos; e atuavam em instituições públicas e privadas de saúde, maternidades e consultórios médicos. Todos os voluntários leram e assinaram o Termo de Consentimento Livre e Esclarecido (TCLE), aprovado pelo Comitê de Ética em Pesquisa (CEP).

\section{Coleta dos dados}

Para a coleta dos dados foi elaborado um questionário com 21 questões, por dois médicos obstetras autores deste estudo, cujo atributo central foi avaliar o nível de conhecimento dos profissionais sobre a utilização do AF na prevenção dos DFTN, sendo que as questões de número um a sete contemplam a descrição das características demográficas dos profissionais (Quadro 1).

Os procedimentos adotados para a coleta dos dados foram: a) os voluntários da pesquisa foram convidados pelo pesquisador a participar de um estudo, sendo informados de que responderiam a um questionário sobre um tema relacionado à sua área de atuação, porém, sem que soubessem, previamente, o tema abordado; b) no momento em que o questionário era entregue ao voluntário, o mesmo era informado, pelo pesquisador ou colaborador, sobre o tema e os objetivos da pesquisa, e, em seguida, se de acordo, assinava o TCLE; c) o voluntário era informado de que suas respostas não poderiam conter qualquer tipo de identificação, nem mesmo para o pesquisador, devendo estas serem respondidas em total privacidade; d) o tempo máximo para o preenchimento do instrumento foi de $10 \mathrm{minu}$ tos. Durante este tempo o pesquisador aguardava, à distância, sem participar do processo; e) ao término do preenchimento, o questionário era introduzido, pelo voluntário em uma urna lacrada. Somente depois de concluída toda a coleta de dados, as urnas lacradas foram abertas pelo pesquisador.

\section{Procedimento de análise dos dados}

O nível de conhecimento foi avaliado por dois enfoques, sendo um baseado no que preconizam as EC sobre a utilização do AF na prevenção dos DFTN $^{5,7,8,13}$ e o outro no que recomenda o $\mathrm{MS}^{14,15}$. Isto posto, foi desenvolvido uma escala de pontuação para cada resposta levando em consideração a importância de cada pergunta dentro do instrumento. Os valores atribuídos para cada variável avaliada (pergunta) foram validados por 
Quadro 1. Formulário para coleta dos dados.

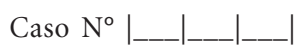

1. Idade: |_____ anos

2. Sexo: [1] Feminino [2] Masculino

3. Profissional: [1] Médico(a) [2] Enfermeiro(a)

4. Ano de graduação: |_____________

5. Unidade de Atendimento: [1] Setor público [2] Setor privado [3] Ambos

6. Nível de formação: [1] Graduação [2] Médico residente [3] Especialização/Residência [4] Mestrado

[5] Doutorado [6] Pós-Doutorado

7. Há quanto tempo realiza atendimento pré-natal? [1] Menos de 01 ano [2] 01 a 02 anos

[3] 02 a 03 anos [4] 03 a 04 anos [5] Mais de 04 anos

8. Prescreve regularmente ácido fólico para gestantes? [1] Sim [2] Não

9. Numa escala de zero a dez, como você avalia seu conhecimento sobre a importância do ácido fólico?

|_-_-___

10. Quais as doenças durante a gestação possíveis de serem prevenidas com o uso do ácido fólico?

( ) Não sei.

11. Já ouviu falar em Defeitos de Fechamento do Tubo Neural (DFTN)? [1] Sim [2] Não

12. Numa escala de zero a dez, como você avalia o seu grau de conhecimento sobre os DFTN?

$\mid$

13. Conhece o papel do ácido fólico na prevenção dos DFTN? [1] Sim [2] Não

14. Você já recebeu capacitação sobre o assunto? [1] Sim [2] Não

15. Caso tenha recebido capacitação sobre o assunto, qual foi a fonte?

16. Qual porcentagem de casos de DFTN pode ser prevenida com a utilização do ácido fólico? [1] Menos $10 \%$ [2] de 11 a $20 \%$ [3] de 21 a $30 \%$ [4] de 31 a $40 \%$ [5] de 41 a $50 \%$ [6] de 51 a $60 \%$ [7] acima de 70\% [8] Não sei

17. Em que período deve ser iniciada a administração do ácido fólico para a prevenção dos DFTN?

( ) Não sei.

18. O ácido fólico deve ser administrado por quanto tempo?

19. Qual dose deve ser administrada por dia? ___ _ não sei.

20. Nas mulheres com gestação anterior, em que o feto que apresentou DFTN, a dosagem do ácido fólico deve ser alterada? [1] Sim [2] Não

21. Se alterada, qual a dose de ácido fólico que deverá ser utilizada por dia? ____mg/dia （ ） Não sei .

dois especialistas independentes e em sistema de revisão por pares (Quadro 2). As perguntas de 1 a 7, 9, 12, 14 e 15 não estão relacionadas com o conhecimento em questão e sim com as características demográficas do entrevistado, bem como a autoavaliação de seu conhecimento sobre o assunto, daí não serem pontuadas. Em adendo, o nível de conhecimento dos profissionais investigados foi autoavaliado por meio da pergunta número 9 do questionário (Quadro 1).

\section{Análise estatística}

Inicialmente foi testada a normalidade (Kolmogorov-Smirnov) dos dados validando ou não a utilização da estatística paramétrica. Para a ten- dência central foram calculadas as médias e para a variabilidade foram utilizados, desvio-padrão e erro padrão da média, respectivamente, nas análises descritivas e inferenciais. Foi realizada a comparação para verificar se havia diferenças entre os níveis de conhecimento, utilizando o teste-t emparelhado e não emparelhado e oneway Anova seguido de comparações post hoc de Bonferroni, quando apropriado, ou teste de Wilcoxon / Man Whitney e o teste de Kruskal Wallis, para as análises paramétricas e não paramétricas, respectivamente. Para todas as análises foram considerados 5\% como nível de significância e utilizamos o software SPSS (versão 17, SPSS, Chicago). 
Quadro 2. Critérios de pontuação para avaliação do conhecimento dos profissionais em função das EC e das recomendações do MS.

\begin{tabular}{|c|c|c|c|}
\hline \multirow[b]{2}{*}{$\begin{array}{l}\text { No da } \\
\text { questão }\end{array}$} & \multirow[b]{2}{*}{ Variável } & \multicolumn{2}{|c|}{ Critérios de pontuação } \\
\hline & & $\begin{array}{c}\text { Em função das evidências } \\
\text { científicas }\end{array}$ & $\begin{array}{l}\text { Em função das } \\
\text { recomendações do } \\
\text { Ministério da Saúde }\end{array}$ \\
\hline 8 & $\begin{array}{l}\text { Prescreve regularmente AF } \\
\text { para gestantes? }\end{array}$ & $\begin{array}{l}\text { Resposta = NÃO, a nota total } \\
\text { do instrumento será ZERO, } \\
\text { por considerarmos que o } \\
\text { questionado não possui } \\
\text { conhecimento sobre o tema. }\end{array}$ & $\begin{array}{l}\text { Resposta = NÃO, a nota total } \\
\text { do instrumento será ZERO, } \\
\text { por considerarmos que o } \\
\text { questionado não possui } \\
\text { conhecimento sobre o tema. }\end{array}$ \\
\hline 10 & $\begin{array}{l}\text { Quais doenças durante a } \\
\text { gestação possíveis de serem } \\
\text { prevenidas com o uso do } \\
\text { AF? }\end{array}$ & $\begin{array}{l}\text { Caso a resposta aberta seja } \\
\text { Dfeitos do Tubo Neural } \\
\text { (DFTN), Mielomeningocele } \\
\text { (MMC) ou Espinha Bífica } \\
\text { consideramos a resposta } \\
\text { correta }=\mathbf{0 , 8} \text { pontos }\end{array}$ & $\begin{array}{l}\text { Caso a resposta aberta seja: } \\
\text { Dfeitos do Tubo Neural } \\
\text { (DFTN), Mielomeningocele } \\
\text { (MMC) ou Espinha Bífica } \\
\text { consideramos a resposta } \\
\text { correta }=\mathbf{0 , 8} \text { pontos }\end{array}$ \\
\hline 11 & $\begin{array}{l}\text { Já ouviu falar em Defeitos } \\
\text { de Fechamento do Tubo } \\
\text { Neural (DFTN)? }\end{array}$ & $\begin{array}{l}\text { Resposta = NÃO, a nota total } \\
\text { do instrumento será ZERO, } \\
\text { por considerarmos que o } \\
\text { questionado não possui } \\
\text { conhecimento sobre o tema. }\end{array}$ & $\begin{array}{l}\text { Resposta = NÃO, a nota total } \\
\text { do instrumento será ZERO, } \\
\text { por considerarmos que o } \\
\text { questionado não possui } \\
\text { conhecimento sobre o tema. }\end{array}$ \\
\hline 13 & $\begin{array}{l}\text { Conhece o papel do AF } \\
\text { na prevenção dos DFTN? }\end{array}$ & $\begin{array}{l}\mathrm{SIM}=\mathbf{0 , 8} \text { pontos } \\
\mathrm{NA} O=\text { zero }\end{array}$ & $\begin{array}{l}\mathrm{SIM}=\mathbf{0 , 8} \text { pontos } \\
\mathrm{NA} O=\text { zero }\end{array}$ \\
\hline 16 & $\begin{array}{l}\text { Que porcentagem de casos } \\
\text { de DFTN podemos prevenir } \\
\text { com a utilização do AF? }\end{array}$ & $\begin{array}{l}\text { de } 50-60 \%=\mathbf{0 , 8} \text { pontos } \\
\text { Outras respostas }=\text { zero }\end{array}$ & $\begin{array}{l}\text { de } 50-60 \%=\mathbf{0 , 8} \text { pontos } \\
\text { Outras respostas }=\text { zero }\end{array}$ \\
\hline 17 & $\begin{array}{l}\text { Em que período deve ser } \\
\text { iniciada a administração do } \\
\text { AF para a prevenção dos } \\
\text { DFTN? }\end{array}$ & $\begin{array}{l}\text { Caso a resposta aberta seja: } \\
\text { pelo menos um mês antes da } \\
\text { concepção, consideramos a } \\
\text { resposta correta }=\mathbf{2} \text { pontos } \\
\text { Outras respostas }=\text { zero }\end{array}$ & $\begin{array}{l}\text { Caso a resposta aberta seja: } \\
\text { de } 60 \text { a } 90 \text { dias antes da } \\
\text { concepção, consideramos a } \\
\text { resposta correta }=2 \text { pontos } \\
\text { Outras respostas }=\text { zero }\end{array}$ \\
\hline 18 & $\begin{array}{l}\text { O AF deve ser administrado } \\
\text { por quanto tempo? }\end{array}$ & $\begin{array}{l}\text { Caso a resposta aberta seja: } 1^{\circ} \\
\text { trimestre, consideramos a } \\
\text { resposta correta }=2 \text { pontos } \\
\text { Outras respostas }=\text { zero }\end{array}$ & $\begin{array}{l}\text { Caso a resposta aberta seja: } \\
\text { durante toda a gestação, } \\
\text { consideramos a resposta } \\
\text { correta }=2 \text { pontos } \\
\text { Outras respostas = zero }\end{array}$ \\
\hline 19 & $\begin{array}{l}\text { Qual dose deve ser } \\
\text { administrada por dia? }\end{array}$ & $\begin{array}{l}\text { Caso a resposta aberta seja: } \\
0.4 \mathrm{mg} / \mathrm{dia}, \text { consideramos a } \\
\text { resposta correta }=2 \text { pontos } \\
\text { Outras respostas }=\text { zero }\end{array}$ & $\begin{array}{l}\text { Caso a resposta aberta seja: } \\
5 \mathrm{mg} / \mathrm{dia} \text {, consideramos a } \\
\text { resposta correta }=2 \text { pontos } \\
\text { Outras respostas }=\text { zero }\end{array}$ \\
\hline 20 & $\begin{array}{l}\text { Nas mulheres com gestação } \\
\text { anterior, em que o feto } \\
\text { apresentou DFTN, a } \\
\text { dosagem do AF deve ser } \\
\text { alterada? }\end{array}$ & $\begin{array}{l}\mathrm{SIM}=\mathbf{0 , 8} \text { pontos } \\
\mathrm{NA} O=\text { zero }\end{array}$ & $\begin{array}{l}\mathrm{SIM}=\mathbf{0 , 8} \text { pontos } \\
\mathrm{N} \tilde{\mathrm{A} O}=\text { zero }\end{array}$ \\
\hline 21 & $\begin{array}{l}\text { Caso sim, qual a dose de AF } \\
\text { que deverá ser utilizada por } \\
\text { dia? }\end{array}$ & $\begin{array}{l}\text { Caso a resposta aberta seja: } 4 \\
\text { mg/dia, consideramos a } \\
\text { resposta correta }=\mathbf{0}, \mathbf{8} \text { pontos } \\
\text { Outras respostas }=\text { zero }\end{array}$ & $\begin{array}{l}\text { Caso a resposta aberta seja: } \\
5 \mathrm{mg} / \mathrm{dia} \text {, consideramos a } \\
\text { resposta correta }=\mathbf{0 , 8} \text { pontos } \\
\text { Outras respostas }=\text { zero }\end{array}$ \\
\hline
\end{tabular}




\section{Resultados}

Os resultados descritivos das variáveis analisadas assim como as características demográficas estão apresentados na Tabela 1.

O nível de conhecimento, dado pela pontuação obtida no questionário, baseado em EC e nas recomendações do MS foram diferentes (4,64 $\pm 0,20$ vs $5,55 \pm 0,15 ; \mathrm{p}<0,001 ;$ Média $\pm \mathrm{EPM})$ (Gráfico 1).

Os participantes do estudo, ao responderem a pergunta nove do Quadro 1, apresentaram um conhecimento relatado médio acerca do assunto de 6,76 $\pm 0,18$ (média \pm EPM). Em adendo, houve diferença significativa entre o nível de conhecimento relatado (nota dada na autoavaliação) e o baseado nas EC $(6,76 \pm 0,18 v s 4,64 \pm 0,15 ; \mathrm{p}<$ 0,001; Média \pm EPM), assim como foi encontrada diferença entre o nível de conhecimento relatado e baseado nas recomendações do MS (6,76 $\pm 0,18$ vs 5,55 $\pm 0,20 ; \mathrm{p}<0,001 ;$ Média $\pm \mathrm{EPM})$ (Gráfico 2). Contudo, o nível de formação não

Tabela 1. Características demográficas.

\begin{tabular}{|c|c|c|}
\hline Variáveis & $\mathbf{n}$ & $\%$ \\
\hline \multicolumn{3}{|l|}{ Gênero $(\mathrm{n}=116)$} \\
\hline Masculino & 46 & 39,6 \\
\hline Feminino & 70 & 60,4 \\
\hline \multicolumn{3}{|l|}{ Profissionais $(\mathrm{n}=118)$} \\
\hline Médico & 95 & 80,5 \\
\hline Enfermeiros & 23 & 19,5 \\
\hline \multicolumn{3}{|l|}{ Local de trabalho $(\mathrm{n}=116)$} \\
\hline Setor Público & 34 & 29,3 \\
\hline Setor Privado & 11 & 9,5 \\
\hline Ambos & 71 & 61,2 \\
\hline \multicolumn{3}{|l|}{ Nível de formação $(\mathrm{n}=118)$} \\
\hline Graduação & 6 & 5 \\
\hline Residência em andamento & 15 & 12,7 \\
\hline Especialização/residência concluídas & 85 & 72 \\
\hline Mestrado & 5 & 4,2 \\
\hline Doutorado & 7 & 5,9 \\
\hline \multicolumn{3}{|l|}{ Tempo que realiza Pré-natal $(\mathrm{n}=118)$} \\
\hline até 4 anos & 27 & 22,9 \\
\hline mais de 4 anos & 91 & 77,1 \\
\hline \multicolumn{3}{|l|}{$\begin{array}{l}\text { Conhece o papel do AF na prevenção } \\
\text { dos DFTN? }(\mathrm{n}=118)\end{array}$} \\
\hline Sim & 111 & 94,1 \\
\hline Não & 7 & 5,9 \\
\hline \multicolumn{3}{|l|}{$\begin{array}{l}\text { Você já recebeu capacitação sobre o } \\
\text { assunto? }(\mathrm{n}=118)\end{array}$} \\
\hline Sim & 14 & 11,9 \\
\hline Não & 104 & 88,1 \\
\hline
\end{tabular}

influenciou o conhecimento dos profissionais no que se refere ao assunto investigado, tanto baseado nas EC quanto no MS ( $\mathrm{p}=0,77$ e $\mathrm{p}=0,97$, respectivamente).

O tempo que os profissionais realizam atendimento pré-natal parece não influenciar no nível de conhecimento baseado em EC e nas recomendações do MS $(4,40 \pm 0,31$ vs $4,71 \pm 0,17 ; p=0,65$, e $5,73 \pm 0,23$ vs $4,93 \pm 0,34 ; p=0,26$, respectivamente). Além disso, não houve diferença entre o nível de conhecimento baseado em EC em função do profissional entrevistado (Médico ou Enfermeiro), assim como para as recomendações do MS $(4,74 \pm 0,17$ vs $4,21 \pm 0,31 ; p=0,17$, e $5,56 \pm$ 0,23 vs $5,50 \pm 0,41 ; \mathrm{p}=0,92$, respectivamente).

\section{Discussão}

Diversos grupos de pesquisa demonstram que a suplementação de AF periconcepcional e durante todo o primeiro trimestre da gravidez, reduz em 50 a 70\% o risco de ocorrência e recorrência dos DFTN $^{3,8,16-20}$. Mesmo sendo extremamente importante a suplementação com AF para a prevenção dos DFTN foi observado que existem algumas divergências entre o que preconizam as EC e as recomendações do MS, em função do período

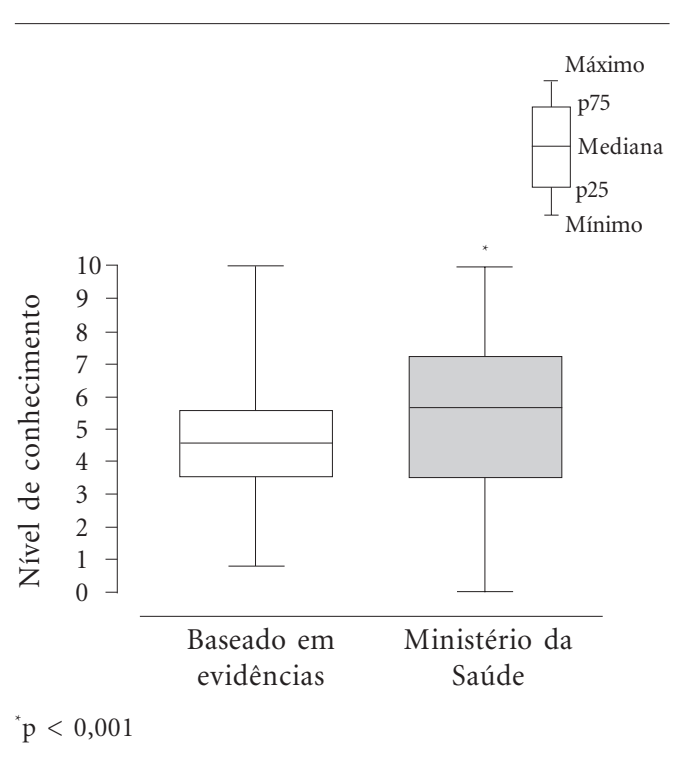

Gráfico 1. Nível de conhecimento dos profissionais de saúde, baseado em EC e nas recomendações do MS. 


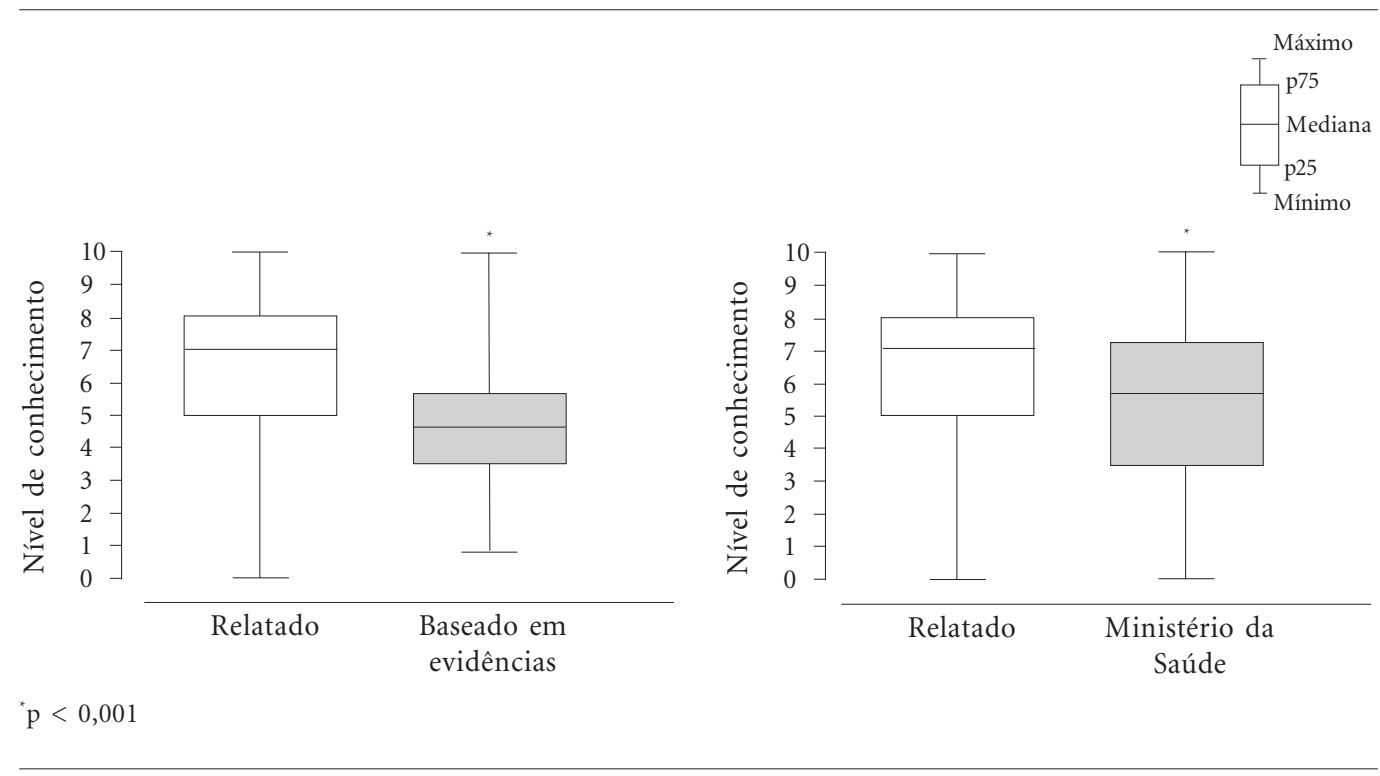

Gráfico 2. Nível de conhecimento relatado versus o baseado em evidências e nas recomendações do Ministério da Saúde.

de início, dosagem ideal e o tempo de utilização do AF na prevenção dos DFTN ${ }^{5,7-11,13-15}$. Em seu Manual Técnico de Pré-Natal e Puerpério ${ }^{14}$ - o MS preconiza uma dose de $5 \mathrm{mg} /$ dia de AF para prevenção dos DFTN enquanto a grande maioria das EC determina que essa dosagem seja $0,4 \mathrm{mg} /$ $\mathrm{dia}^{5,7,8,13}$. Cabe destacar que, nesse mesmo documento, o MS atesta que a referida dose poderia, quando associada ao sulfato ferroso, prevenir a anemia, justificando, talvez, a divergência entre os valores recomendados e as EC, permanecendo assim um óbice na temática.

Os profissionais que participaram do estudo demonstraram maior nível de conhecimento baseado nas recomendações do MS. Provavelmente, isso se deve à formação acadêmica desses profissionais que enfatizam, prioritariamente, as diretrizes dos manuais do MS em detrimento das EC, que necessitam de acesso a bases de dados especializadas, quase sempre apresentadas no idioma inglês. Essa busca da informação científica, muitas vezes é dificultada pela falta de infraestrutura das escolas ou, talvez, pela deficiência no currículo de algumas instituições que não mantêm em sua grade curricular a disciplina que permeia a tomada de decisão baseada em evidências como, por exemplo, a Metodologia da Pesquisa Científica. Além disso, os resultados demonstram que o nível de conhecimento dos participantes do estudo quanto à prevenção dos DFTN utilizando a suplementação de AF é baixo, corroborando os resultados de outros dois estudos ${ }^{21,22}$ realizados anteriormente com o mesmo objetivo por outros grupos de pesquisa, que também evidenciaram a falta de conhecimento dos profissionais de saúde frente ao assunto investigado.

$\mathrm{O}$ conhecimento relatado pelos participantes do estudo foi maior ao que realmente foi avaliado tanto em função das EC quanto nas recomendações do MS, demonstrando, dessa forma, que os profissionais superestimam seus conhecimentos em relação à utilização do AF na prevenção do DFTN. No presente estudo, a formação dos participantes não influenciou no nível de conhecimento baseado em EC e nem nas recomendações do MS. Contudo, os profissionais com doutorado apresentaram maior média no nível de conhecimento baseado em EC, provavelmente devido à maior facilidade de acesso às EC (média $=5,1)$ e a maior independência investigadora.

Não foi observado neste estudo diferença entre o tempo em que o profissional realiza prénatal e os níveis de conhecimento baseados em EC e no MS, ou seja, o tempo que o profissional realiza a atividade de atendimento às gestantes parece não ser um fator determinante para um maior conhecimento do tema investigado. Estes achados corroboram a impressão de outros estudos, como o realizado por Abu-Hammad et al. ${ }^{21}$, no qual os profissionais com mais de 15 anos de formados não possuíam maior conhecimento sobre a utilização de AF. Em contrapar- 
tida, Miranda et al. ${ }^{22}$ demonstraram em um estudo realizado em dois hospitais de Porto Rico, sendo um público e o outro privado, com médicos obstetras, pediatras e clínicos gerais, que quanto maior o tempo de profissão maior o conhecimento acerca da administração do AF na prevenção do DFTN. Cabe ressaltar, que os profissionais médicos que apresentaram maior conhecimento trabalhavam no hospital público que naquele país é mais rigoroso na implementação de políticas governamentais, posto que a instituição é utilizada para treinamento de outros profissionais.

Quando comparado o conhecimento dos médicos e enfermeiros não foram observadas diferenças, provavelmente, porque as recomendações governamentais para a condução do prénatal são as mesmas para todos os envolvidos nesse tipo de assistência. Os dados deste trabalho corroboram os dados de outros dois estu$\operatorname{dos}^{21,22}$ que abordaram diretamente a mesma temática e não encontraram diferença entre o nível de conhecimento sobre a utilização do AF para a prevenção dos DFTN entre diferentes especialidades médicas.

Os resultados deste estudo demonstraram que $94,1 \%$ dos avaliados relataram possuir conhecimento sobre o assunto, resultados estes semelhantes a outro estudo conduzido por grupo israelense, no qual 93\% dos entrevistados relatam conhecer o papel do AF na prevenção dos DFTN ${ }^{21}$. Não obstante, neste estudo, apenas 11,9\% afirmaram já ter recebido algum tipo de capacitação sobre o assunto e 73,6\% erraram ao relatar não ser necessário aumentar a dose do AF em mulheres com antecedentes de DFTN, divergindo das $\mathrm{EC}^{23}$ que preconizam o aumento da dose nestes casos. Quando questionados sobre o período de início da sua utilização, 64,2\% não sabiam ou erraram a resposta, sendo que $33,8 \%$ responderam que o período ideal para o inicio da suplementação é de 60 a 90 dias antes da concepção, como recomendado pelo MS e apenas $2 \%$ responderam segundo as EC. Essas respostas demonstram que existe um desconhecimento da maioria dos profissionais sobre a época do fechamento do tubo neural que ocorre, aproximadamente, entre $22^{\circ} \mathrm{e}$ o $28^{\circ}$ dia. Esses índices foram superiores aos encontrados em profissionais israelenses $(53 \%)^{21}$. Contudo, neste estudo, os profissionais responderam ao questionário em suas residências, o que possibilitou a consulta de fontes bibliográficas, $\mathrm{o}$ que pode ter interferido sobremaneira nos resultados do estudo, além de a amostra ser constituída apenas por médicos. Ao investigar a dose ade- quada de AF, 68,6\% dos participantes responderam $5 \mathrm{mg} /$ dia, conforme recomendação do MS, sendo que, 28\% não sabiam ou erraram a dose/ dia recomendada, valores esses inferiores aos obtidos no estudo realizado em Porto Rico, no qual $62 \%$ dos médicos avaliados não sabiam qual a dose correta para a suplementação com $\mathrm{AF}^{22}$. Quanto ao tempo de utilização do AF, 68,6\% responderam que deve ser administrado no primeiro trimestre da gestação, conforme as EC. Nessa questão, prevaleceu o conhecimento baseado em EC, diferentemente do observado nas respostas anteriores.

Diante desses resultados que avaliaram o conhecimento real e o conhecimento relatado pelos profissionais investigados, foi constatado que apesar dos participantes afirmarem que conhecem o papel do AF na prevenção dos DFTN, isto não foi demonstrado pelos resultados deste estudo, assim como os outros estudos que investigaram o conhecimento de médicos sobre a utilização de $A^{21,22}$. Foi observado também que há prevalecimento do conhecimento baseado nas recomendações do MS, em detrimento daquele baseado nas EC. Além disso, algumas deficiências graves foram observadas no conhecimento dos profissionais como, por exemplo, o período de início da suplementação do AF. Os resultados também apontam que a falta de capacitação profissional sobre o assunto pode ser uma das principais causas dessa grande diferença de conhecimento relatado em relação ao conhecimento avaliado. Uma possível limitação do presente estudo foi o fato de não serem avaliados médicos do programa de saúde da família que realizam prénatal de baixo risco. Cabe ainda ressaltar que, quanto à questão da mudança na dosagem do AF em pacientes com histórico de DFTN, de acordo com as evidências encontradas na literatura médica, essa dose deve ser aumentada 10 vezes, porém como o ministério da saúde já indica uma dose bastante elevada, realmente não existe necessidade em alterar a dose. Fato esse que pode ser observado na interpretação das questões 19 , 20 e 21 do instrumento utilizado no estudo.

Os resultados obtidos aqui indicam a necessidade de práticas de políticas públicas direcionadas à capacitação permanente dos profissionais envolvidos no atendimento à mulher. Considerando-se que mais da metade das gestações não são planejadas, como encontrado em estudo realizado em nosso núcleo de pesquisa com gestantes atendidas na rede pública, destacamos a importância fundamental de esclarecer à população quanto à necessidade da utilização do 
ácido fólico na prevenção dos DFTN. Alertando que a prevenção, para ser efetiva, deve ser iniciada no período pré-concepcional e prolongar-se até o término do primeiro trimestre de gestação.

Considerando os elevados custos financeiros relacionados ao tratamento e acompanhamento dos pacientes com DFTN, além das graves consequências pessoais, familiares e sociais, contrapondo-se à eficiência e ao baixo custo da prevenção, esperamos que este trabalho possa, de forma efetiva, contribuir para a elaboração de estratégias de saúde pública, que interfiram diretamente na prevenção dessas graves anomalias congênitas.

\section{Conclusão}

Embora a grande maioria dos profissionais avaliados tenha relatado conhecer o papel do AF na prevenção dos DFTN, existe importante nível de desconhecimento de médicos e enfermeiros obstetras sobre a utilização do AF quanto ao tempo de utilização, o inicio da suplementação e a dosagem ideal, pontos relevantes na prevenção dos DFTN. Em adendo, o nível de formação, a profissão e o tempo de experiência em atendimento pré-natal não influenciaram o conhecimento sobre o papel do AF na prevenção do DFTN. Portanto, são necessárias políticas públicas que objetivam a capacitação dos profissionais que atuam no atendimento da mulher para minimizar os riscos de tais anomalias.

\section{Colaboradores}

RC Conceição, MAH Barbosa, LL Dornela, PS Ramos, DS Castellano Filho, DR Ricardo, AA Calado e JM Bastos Netto participaram igualmente de todas as etapas de elaboração do artigo. 


\section{Referências}

1. Aguiar MJ, Campos AS, Aguiar RA, Lana AM, Magalhaes RL, Babeto LT. Neural tube defects and associated factors in liveborn and stillborn infants. J Pediatr 2003; 79(2):129-134.

2. Koren G, Goh I. Increasing folate supplementation for selected groups of Canadian women. J Obstet Gynaecol Can 2007; 29(1):992-996.

3. Botto LD, Moore CA, Khoury MJ, Erickson JD. Neural-tube defects. N Engl J Med 1999; 341(20): 1509-1519.

4. Melvin EC, George TM, Worley G, Franklin A, Mackey J, Viles K, Shah N, Drake CR, Enterline DS, McLone D, Nye J, Oakes WJ, McLaughlin C, Walker ML, Peterson P, Brei T, Buran C, Aben J, Ohm B, Bermans I, Qumsiyeh M, Vance J, Pericak-Vance MA, Speer MC. Genetic studies in neural tube defects. NTD Collaborative Group. Pediatr Neurosurg 2000; 32(1):1-9.

5. Center of Desease Control. Centers for Disease Control and Prevention. Preventing Neural Tube Birth Defects: A Prevention Model and Resource Guide. $4^{\text {th }}$ ed. Atlanta: Centers for Disease Control and Prevention; 2002.

6. Locksmith GJ, Duff P. Preventing neural tube defects: the importance of periconceptional folic acid supplements. Obstet Gynecol 1998; 91(6):1027-1034.

7. Kondo A, Kamihira O, Ozawa H. Neural tube defects: prevalence, etiology and prevention. Int J Urol 2009; 16(1):49-57.

8. United State Preventive Services Task Force. Folic acid for the prevention of neural tube defects: U.S. Preventive Services Task Force recommendation statement. Ann Intern Med 2009; 150(9):626-631.

9. American Academy of Family Physicians. Summary of Recommendations for Clinical and Preventive Services. 2008. [serial on the Internet]. [acessado 2008 set 18]. Disponível em: www.aafp.org/online/ en/home/clinical/exam.html

10. ACOG Committee on Practice Bulletins. Clinical management guidelines for obstetrician-gynecologists. Obstet Gynecol 2003; 102(1):203-213.

11. Centers for Disease Control and Prevention (CDC). Use of supplements containing folic acid among women of childbearing age-United States, 2007. Morb Mortal Wkly Rep 2008; 57(1):5-8.

12. Royal College of Obstetricians and Gynaecologists. Periconceptional folic acid and food fortification in the prevention of neural tube defects. SAC Opinion Paper 2003; (4):1-8.

13. Wolff T, Witkop CT, Miller T, Syed SB. Folic acid supplementation for the prevention of neural tube defects: an update of the evidence for the U.S. Preventive Services Task Force. Ann Intern Med 2009; 150(9):632-639.
14. Brasil. Ministério da Saúde (MS). Secretaria de Atenção à Saúde. Departamento de Ações Programáticas Estratégicas. Manual Técnico - Pré-Natal e Puerpério - Atenção Qualificada e Humanizada. Brasília: MS; 2006.

15. Federação Brasileira das Associações de Ginecologia e Obstretícia (Febrasgo). Manual de Orientação - Assistência Pré-Natal. Rio de Janeiro: Febrasgo Editora; 2007.

16. Berry RJ, Li Z, Erickson JD, Li S, Moore CA, Wang H, Mulinare J, Zhao P, Wong LY, Gindler J, Hong SX, Correa A. Prevention of neural-tube defects with folic acid in China. China-U.S. Collaborative Project for Neural Tube Defect Prevention. N Engl J Med 1999; 341(20):1485-1490.

17. Czeizel AE, Dobo M, Vargha P. Hungarian cohortcontrolled trial of periconceptional multivitamin supplementation shows a reduction in certain congenital abnormalities. Birth Defects Res A Clin Mol Teratol 2004; 70(11):853-861.

18. De Wals P, Tairou F, Van Allen MI, Uh SH, Lowry RB, Sibbald B, Evans JA, Van den Hof MC, Zimmer P, Crowley M, Fernandez B, Lee NS, Niyonsenga T. Reduction in neural-tube defects after folic acid fortification in Canada. N Engl J Med 2007; 357(2): 135-142.

19. Goh YI, Bollano E, Einarson TR, Koren G. Prenatal multivitamin supplementation and rates of congenital anomalies: a meta-analysis. J Obstet Gynaecol Can 2006; 28(8):680-689.

20. Lumley J, Watson L, Watson M, Bower C. Periconceptional supplementation with folate and/or multivitamins for preventing neural tube defects. Cochrane Database Syst Rev 2001; (4):CD001056.

21. Abu-Hammad T, Dreiher J, Vardy DA, Cohen AD. Physicians' knowledge and attitudes regarding periconceptional folic acid supplementation: a survey in Southern Israel. Med Sci Monit 2008; 14(5):CR262267.

22. Miranda A, Davila Torres RR, Gorrin Peralta JJ, Montes de Longo I. Puerto Rican primary physicians' knowledge about folic acid supplementation for the prevention of neural tube defects. Birth Defects Res A Clin Mol Teratol 2003; 67(12):971-973.

23. Grosse SD, Collins JS. Folic acid supplementation and neural tube defect recurrence prevention. Birth Defects Res A Clin Mol Teratol 2007; 79(11):737-742.

Artigo apresentado em 11/07/2011

Aprovado em 30/08/2011

Versão final em 09/09/2011 
